\title{
Stregoneria e utopia. Luciano Parinetto vent'anni dopo
}

\author{
di Manuele Bellini
}

manuele.bellini@gmail.com

\begin{abstract}
Twenty years after his death, the reflections of Luciano Parinetto (1934-2001), who was associate professor of Moral Philosophy at the University of Milan, remain, on the one hand, on the relationship between witchcraft and diversity, and on the other hand, on the value of utopian hope. Despite the fact that the course of history has preferred the path of integration to that of revolution, dialectics remains the picklock to criticize alienated social situations.
\end{abstract}

Keywords: Revolutionary Dialectic, Utopian Hope, Diversity, Witchcraft

\section{Una "damnatio memoriae"?}

Affrontare il rapporto tra stregoneria e utopia vuol dire considerare de facto l'intero pensiero di Parinetto: chiarire, infatti, la relazione e, in via preliminare, il significato dei concetti sopra adoperati, che di certo richiedono una precisa contestualizzazione storica e, in modo correlato, un'altrettanto puntuale individuazione semantica, significa addentrarsi nella complessità irriducibile della filosofia parinettiana, per sua natura antisistematica, e spaziare sincronicamente tra le varie tematiche che a questi stessi concetti si agganciano spesso in modo imprescindibile.

Tale impresa si rende, d'altronde, opportuna dal momento che la figura e l'opera di Parinetto, nonostante siano trascorsi vent'anni dalla sua morte, avvenuta a Chiari (BS) il 22 dicembre 2001, sono ancora troppo poco noti, al di là, beninteso, della cerchia degli allievi universitari che ne hanno 
testimoniato, in più occasioni, la ricchezza e l'attualità 1 . Tuttavia, al di fuori dell'ambiente accademico, la sua figura non ha ancora avuto un meritato riconoscimento e, del resto, anche nell'ambiente universitario, l'evidente assenza di un interesse ${ }^{2}$, anche solo di carattere archivistico-documentario ${ }^{3}$, sembra testimoniare, più che un oblio benché amaro, una vera e propria damnatio memoriae 4 .

Per avere un'idea della scarsa considerazione intellettuale da lui goduta, con qualche eccezione ${ }^{5}$, nel mondo universitario, e della conseguente distrazione nei riguardi dei suoi studi, è utile richiamare quanto scritto da E.I. Rambaldi in un lungo articolo pubblicato negli Annali di Storia delle Università Italiane, nel quale, ricostruendo la storia degli insegnamenti di Filosofia presso l'Università Statale di Milano, fornisce anche sommarie indicazioni sui loro titolari. Così scrive nello specifico: «Luciano Parinetto, allievo di Dal Pra, dopo aver coltivato studi storici (Magia e ragione. Una polemica sulle streghe in Italia intorno al 1750, 1974) ebbe una seconda fase di pensiero, nella quale insegnò Filosofia morale (associato nel 1983; lasciò l'insegnamento nel 1999) ed espresse posizioni via via più radicali che

\footnotetext{
1 Tra l'altro, nei contributi raccolti in M. Bellini (a cura di), Corpo e rivoluzione. Sulla filosofia di Luciano Parinetto, Mimesis, Milano-Udine 2012 e negli atti del Convegno di cui alla nota 2. 2 Dalla sua morte, solo un convegno è stato organizzato in sua memoria (in data 5 novembre 2004 presso l'Università degli Studi di Milano-Bicocca), e, nonostante vi avessero partecipato anche esponenti del mondo universitario come Elio Franzini e Mario Cingoli, fu ideato e realizzato da alcuni suoi fedeli ex allievi, come Nicoletta Poidimani, che ne fu collaboratrice per anni, Mario Domina e Francesco Muraro. Cfr. N. Poidimani (a cura di), Luciano Parinetto. L'utopia di un eretico, Mimesis, Milano 2005.

${ }^{3}$ Cfr. M. Bellini, "Il Fondo Parinetto tra marxismo, stregoneria e alchimia", in F. Formenti (a cura di), La Biblioteca Morcelliana nel bicentenario (1817-2017). Studi e ricerche, Morcelliana, Brescia 2017, pp. 257-262; già in Materiali di Estetica. Terza serie, IV/1, 2017, pp. 484-489.

${ }_{4}^{4}$ Ho tentato di individuarne alcune motivazioni in M. Bellini, Dialettica del diverso. Marxismo e antropologia in Luciano Parinetto, Mimesis, Milano-Udine 2018, in particolare pp. 101-103. Da questo testo riprendo anche alcuni passi, con varianti.

5 Tra cui Elio Franzini (di cui si veda "Utopie", in N. Poidimani, Luciano Parinetto. L'utopia di un eretico, cit., pp. 39-46) e Gabriele Scaramuzza ("Un amore di Parinetto", in Il brutto all'Opera. L'emancipazione del negativo nel teatro di Giuseppe Verdi, Mimesis, Milano-Udine 2013, pp. 157-181).
} 
mischiavano disordinatamente proteste civili, politiche e di costume (Marx diversoperverso, 1996)»6.

Da questa presentazione, che restituisce in parte, e in modo non sottile, l'opinione accademica corrente sul valore delle ricerche di Parinetto, è utile prendere avvio. Con ogni evidenza, si tratta di un'annotazione che, assumendo la forma di un giudizio critico piuttosto liquidatorio, e ciò a prescindere dall'esposizione necessariamente sintetica, pare quanto meno ingenerosa sul piano intellettuale e non condivisibile nel merito.

Prima di tutto Rambaldi sembra avallare l'isolamento intellettuale di Parinetto, non specificando (come fa, per esempio, nel medesimo articolo per Carlo Montaleone), che fu collaboratore per anni di Remo Cantoni, con il quale è lecito supporre avesse in comune interessi antropologici, del resto testimoniabili negli studi sulla stregoneria.

In secondo luogo, ancorché sia indubbia l'impostazione storico-filosofica della sua tesi di laurea, discussa con Dal Pra il 18 novembre 1961, dal titolo Magia e ragione (che diventerà la sua prima monografia, edita nel 1974) 7 , è difficile rinvenire uno iato tra questo testo e i successivi, che altro non fanno che approfondire la tesi in esso sostenuta, sebbene di certo variamente articolata, secondo cui la caccia alle streghe sarebbe, come vedremo, la propaganda ideologica dell'economia capitalistica che demonizza il diverso.

In terzo luogo, v'è da precisare che Parinetto non abbandona, dopo questo testo, gli studi storici, che anzi sono sempre richiamati alla base delle sue ricerche come premesse di fondo per qualunque lavoro interpretativo che non sia subordinato a prese di posizione preconcette sulle realtà di cui tratta. $\mathrm{Ne}$ sono prove opere come Solilunio. Erano donne le streghe?, pubblicato da Pellicani nel 1991, o La rivolta del diavolo - sottotitolo: Müntzer, Lutero e la

\footnotetext{
${ }^{6}$ E.I. Rambaldi, "Gli insegnamenti filosofici nella Facoltà di Lettere (1924-1968)", in E. Brambilla, M. G. di Renzo Villata (a cura di), Per una storia dell'Università di Milano, CLUEB, Bologna 2008, p. 123.

${ }^{7}$ Cfr. L. Parinetto, Magia e ragione. Una polemica sulle streghe in Italia intorno al 1750, La Nuova Italia, Firenze 1974 (riedito col titolo I Lumi e le streghe, Colibrì, Milano 1998).
} 
rivolta dei contadini in Germania - edito da Rusconi nel 1999. Tuttavia Parinetto sa che la filosofia non si riduce alla sua storia e interpretazioni storicistiche o filologiche, quando esclusive, corrono il rischio di confinare i fenomeni culturali nel loro tempo, senza permettere di coglierne il senso per l'oggi: ne è una prova il commento ai frammenti di Eraclito, dove più volte ironizza su quegli «ipercritici» che, incontrando termini presuntivamente non ancora in uso nel V secolo a.C., sospettano dell'autenticità delle fonti ricorrendo a «balletti filologal-interpretativi», che inchiodano la lettura dell'autore alle lacune documentarie senza prendere atto che l'argumentum ex silentio paralizza letture attualizzanti ${ }^{8}$.

In quarto luogo va aggiunto che Marx diversoperverso, che è del 1996, non dà avvio né mette capo a alcuna seconda fase della filosofia parinettiana dal momento che è solo la riedizione, tra l'altro, di Né dio né capitale ${ }^{9}$ e di Corpo e rivoluzione in Marx ${ }^{10}$, che a loro volta raccolgono saggi pubblicati nei primi anni Settanta sulla rivista "Utopia», di cui Parinetto era anche redattore; tutti testi scritti, dunque, tra Magia e ragione e le altre monografie sulla stregoneria elaborate negli anni Ottanta e Novanta ${ }^{11}$, le quali, peraltro, indagano lo sfondo storico-sociale dei temi affrontati in alcuni di questi stessi contributi.

Non da ultimo, definire «disordinato» il suo approccio metodologico, benché opinione legittima, non tiene conto dell'impostazione dichiaratamente antisistematica e antiorganica della sua scrittura: nell'avvertenza a Corpo e rivoluzione in Marx, Parinetto scrive che, «come il corpo di Penteo, sbranato

\footnotetext{
${ }^{8}$ Cfr. Eraclito, Fuoco non fuoco. Tutti i frammenti del filosofo di Efeso, a cura di L. Parinetto, Mimesis, Milano 1994, pp. 35, 36, 46, 50, 55, 57, 74, 81, 165, 198.

9 Cfr. L. Parinetto, Né dio né capitale. Marx, marxismo, religione, Moizzi Contemporanea, Milano 1976.

${ }_{10}$ Cfr. L. Parinetto, Corpo e rivoluzione in Marx. Morte, diavolo, analità (1977), Mimesis, Milano-Udine 2015.

${ }^{11}$ L. Parinetto, Streghe e politica. Dal rinascimento italiano a Montaigne, da Bodine a Naudé, prefazione di G. Galli, I.P.L., Milano 1983; L. Parinetto, Il ritorno del diavolo, Mimesis, Milano 1996; L. Parinetto, La traversata delle streghe, cit. (tutti raccolti in Streghe e potere. Il capitale e la persecuzione dei diversi, Rusconi, Milano 1998).
} 
e ucciso dalle Baccanti, la tematica fondamentale qui circola, a brani, in tutti i saggi. Sta al lettore, se vuole ricomporre il cadavere, riunificarla a suo modo. Io ho preferito - in odio al sistema e all'identico - il dilaniamento delle membra sparse»12. L'ambizione al "sistema" è ritenuta di per sé mistificatoria nella sottaciuta presunzione esaustiva e nella correlata convinzione che esista una verità interpretativa monolitica, priva di Trennungen problematiche (per usare un termine caro al pensiero dialettico), quando per Parinetto la verità è pluristratificata e metamorfica, e alla maniera lessinghiana, come amava spesso ricordare ${ }^{13}$.

Infine, bollare come radicali le sue posizioni può anche interpretarsi, più che come una critica, come una nota di merito, esprimendo la volontà del pensiero di andare alla radice delle cose; d'altronde, se Rambaldi intende il termine "radicale" nel senso di "estremista", il merito potrebbe addirittura sembrare un elogio, richiamando l'immagine del «furioso» di Bruno (di cui Parinetto traduce il De magia e il De vinculis in genere, oltre a curarne, traducendo anch'essi, gli atti del processo) ${ }^{14}$, il quale, stando sugli estremi, riesce meglio a conoscere e ad apprezzare la natura rispetto al sapiente, che invece, stando nel mezzo, perde la visione del tutto di cui è parte.

\section{Alienazione, dialettica e utopia}

Ma veniamo al percorso intellettuale di Parinetto, che non è privo di qualche significativo ripensamento. Magia e ragione è, si è detto, la rielaborazione e l'ampliamento della tesi di laurea in Filosofia, discussa nel 1961. Di lì a poco, nel 1965, Dal Pra dà alle stampe il fondamentale contributo

\footnotetext{
${ }^{12}$ L. Parinetto, Corpo e rivoluzione in Marx, cit., p. 400.

${ }^{13}$ Cfr. L. Parinetto, Nostra signora dialettica. Unificazione e scissione in Lessing, Herder, Schiller, prefazione di A. Marini, A. Pellicani, Roma 1991, pp. 55-211.

${ }^{14}$ Cfr. G. Bruno, La magia e le ligature, a cura di L. Parinetto, Mimesis, Milano 2000; M. L. Ghezzi (a cura di), Processo e morte di Giordano Bruno. I documenti con un saggio introduttivo di Luciano Parinetto, Milano-Udine 2012 (già edito, senza la curatela di Ghezzi, per i tipi di Rusconi, Milano 1999).
} 
La dialettica in Marx ${ }^{15}$, dove riconduce l'origine della dialettica (della quale non aveva trattato nel corso dell'anno accademico 1958-59, che Parinetto aveva seguito16, e che era più incentrato, come ha scritto Dario Borso, su «un Marx illuminato da Preti») ${ }^{17}$ soprattutto al rovesciamento dei rapporti di predicazione effettuato mediante la critica del "misticismo logico" di Hegel, articolata da parte di Marx sulla scorta della nuova prospettiva antropologica di Feuerbach.

Parinetto, dal canto suo, dopo il testo di Dal Pra, fa stampare, per La Goliardica, una dispensa dal titolo La nozione di alienazione in Hegel, Feuerbach e Marx ${ }^{18}$, relativa a un seminario di Filosofia morale per il corso di Remo Cantoni dell'a.a. 1966-'67: didascalica nell'impianto e tradizionale nei contenuti esplorati, si può collocare in modo coerente nell'alveo degli studi marxisti post-sessantottini incentrati sulla complessità di rapporti tra alienazione e oggettivazione, temi sui quali vertevano in quegli anni anche $\mathrm{i}$ corsi di Franco Fergnani, suo collega alla Statale ${ }^{19}$. Proseguendo su questa linea, negli anni immediatamente seguenti, da un lato, estende i propri studi al riguardo «in due direzioni già abbozzate nel seminario del 1966-'67: il corpo, l'utopia. Il corpo come "materia" e insieme "luogo" di resistenza; l'utopia come "trionfo" in fieri sull'alienazione, come suo "doppio"»"20. Il risultato di queste riflessioni si apprezza nella stesura, dal '71 al '73, di numerosi articoli pubblicati sul mensile marxista di cultura «Utopia»"21, di cui è redattore,

${ }^{15}$ Cfr. M. Dal Pra, La dialettica in Marx. Dagli scritti giovanili all"'Introduzione alla critica dell'economia politica”, Laterza, Bari 1965.

16 Come scrive Dario Borso nella prefazione a L. Parinetto, Teorie dell'alienazione. Hegel, Feuerbach, Marx, ShaKe, Milano 2012, p. 7.

17 D. Borso, introduzione a M. Dal Pra, Il pensiero filosofico di Marx, ShaKe, Milano 2011, p. 11.

18 Cfr. L. Parinetto, La nozione di alienazione in Hegel, Feuerbach e Marx, a cura di M. Lavaggi e M. Pitta, La Goliardica, Milano 1968, 1969², ora riedita, a cura di D. Borso, col titolo Teorie dell'alienazione, cit.

19 Valga come esempio F. Fergnani, Alienazione e oggettivazione nella problematica del pensiero moderno e contemporaneo, Cuem, Milano s.d. ma presumibilmente 1972.

20 D. Borso, "Prefazione", in L. Parinetto, Teorie dell'alienazione, cit., p. 8.

${ }^{21}$ Nello specifico: L. Parinetto, "Sul noviziato feuerbachiano di Marx", luglio 1971, pp. 7-14;

"La religione nel 'Capitale", agosto 1971, pp. 4-10; "Karl Marx e Moses Hess", settembre-

Materiali di Estetica - N. 8.2: 2021, Pagina 417 
alcuni dei quali, sul rapporto tra alienazione e utopia in autori del pensiero illuministico, confluiranno, interamente rivisti, in Nostra signora dialettica del 1991, un titolo di per sé sintomatico, anche se ex post, del corso intellettuale di Parinetto a partire dai primi anni Settanta. Egli arriva infatti a negare l'idea che "quella della dialettica" sia "un'immacolata concezione nella testa di Marx»; le influenze che in essa si ravvisano non sono infatti quelle "che la coiné bibliografica», in cui si può includere in parte il volume di Dal Pra, «considera canoniche nel delineare la formazione di Marx (Feuerbach, Hess etc. etc.)", ma fonti più anteriori, illuministiche, poco o per nulla evidenziate, quali «Rousseau, Diderot, Herder, Lessing, Schiller, Fichte», «per non dire di quelle alchemiche», su cui nella prefazione dichiara di essersi già soffermato altrove ${ }^{22}$ - in particolare, lo precisiamo, in Faust e Marx del 1989 e in Alchimia e utopia del 1990 - anche se qui ne fa continui riferimenti.

Alla luce delle nuove ricerche, si evince come della prospettiva di Dal Pra Parinetto trattenga forse soltanto l'idea, comunque fondamentale, che la dialettica è il nucleo portante del pensiero marxiano. Per il resto, ridimensiona il «noviziato feuerbachiano» del giovane filosofo di Treviri23; altre fonti rimosse sono indagate: vi sono quelle illuministiche e romantiche, s'è detto, ma vi figurano anche le culture popolari quali i costumi delle streghe, o saperi occulti come la tradizione alchemica moderna, la cultura magica umanistico-rinascimentale, per non tacere visioni del mondo ai limiti

ottobre 1971, pp. 11-18; "Sulla forma della religione in Marx", novembre 1971, pp. 7-13; "Tre immagini dell'intellettuale Marx", dicembre 1971, pp. 7-14; "Alienazione e utopia in Rousseau", marzo 1972, pp. 8-16; "Alienazione e utopia in Lessing", maggio 1972, pp. 3-8; parte II, giugno 1972, pp. 8-14; "Antimacchinismo e umanità in Herder", settembre 1972, pp. 3-10; "Ernst Bloch: un teologo?", ottobre 1972, pp. 18-25; "Corpo e rivoluzione in Marx", gennaio 1973, pp. 3-12; "L'ateismo di Marx: una leggenda", marzo 1973, pp. 3-12; "Morte e utopia", giugno 1973, pp. 3-13; "Schiller segreto", settembre 1973, pp. 3-10; "Gioco e rivoluzione in Schiller", ottobre 1973, pp. 3-13; "L'utopia del diavolo. Egualitarismo e transessualità", dicembre 1973, pp. 19-28.

${ }^{22}$ L. Parinetto, Nostra signora dialettica, cit., pp. 4, 5.

${ }^{23}$ Cfr. L. Parinetto, "Sul noviziato feuerbachiano di Marx", cit., raccolto poi in Né dio né capitale, cit., pp. 11-46. 
dell'eresia come la mistica tedesca classica ${ }^{24}$. Scrive al riguardo che «il verbo alienare non giunge a Hegel e a Marx, come qualche teologo ha insinuato, dalle lettere paoline (o non solo da esse), ma, forse, dal vocabolario alchemico/ermetico»; il termine alienare compare infatti in vari testi alchemici che «lo impiegano tecnicamente, per spiegare cioè il divenir altro di un metallo. Alienazione, allora, diventa termine altro per trasmutazione e alienare diventa quindi un termine centrale per l'operare alchemico, che, essendo dialettico molto prima di Marx e Hegel, collega dialettica e alienazione» ${ }^{25}$.

È a questo proposito che si è parlato di «marxismo magico»: lo ha fatto Marcello Veneziani, in un articolo intitolato L"'altra sinistra' resta immaginaria e pubblicato su «Il sabato» in data 7 aprile 1989, dove include in una comunanza di intenti il Faust e Marx parinettiano, Occidente misterioso di Giorgio Galli e Il senso della possibilità. La sinistra e l'immaginario di Attilio Mangano ${ }^{26}$. Tuttavia, a Parinetto non interessa indagare la relazione tra esoterismo e politica, né tantomeno fare della dietrologia: il suo sguardo non è rivolto al passato, ma proiettato nel futuro; non intende scovare le presunte ragioni occulte degli eventi storici che presiedono al fenomeno della stregoneria, ma demistificare i meccanismi di

\footnotetext{
${ }^{24}$ Cfr., per esempio, L. Parinetto, Alchimia e utopia (1990), Mimesis, Milano 2004, e le curatele, tutte edite da Mimesis, di N. Cusano, Il dio nascosto, 1983 (per Marcos y Marcos), $1992^{2}, 2010^{3}$, A. Silesius, L'altro io di dio. 414 epigrammi dal "Viatore cherubico" di Angelus Silesius (Johannes Scheffler), 1993, e J. Bohme, La vita sovrasensibile. Dialogo tra un maestro e un discepolo, 1998.

${ }^{25}$ L. Parinetto, Alchimia e utopia, cit., p. 100.

${ }^{26}$ Cfr. L. Parinetto, Faust e Marx. Metafore alchemiche e critica dell'economia politica. Satura inconclusiva non scientifica (1989), Mimesis, Milano 2004; G. Galli, Occidente misterioso, Rizzoli, Milano 1989 (riedito col titolo Cromwell e Afrodite per Kaos, Milano 1995); A. Mangano, Il senso della possibilità. La sinistra e l'immaginario, A. Pellicani, Roma 1989. Peraltro l'espressione "marxismo magico» è considerata da Mangano «un'operazione "giornalistica" col suo tipico folklore». A. Mangano, "Il pensiero di Luciano Parinetto: un marxismo magico?", in N. Poidimani (a cura di), L'utopia di un eretico, cit., p. 33, opinione condivisa da Galli, che parla di «piccolo scoop giornalistico» inventato ad hoc. Cfr. G. Galli, "Solilunio tra streghe e alchimisti", in ivi, p. 58.
} 
costituzione dello stigma nella prospettiva di un umanismo che valorizzi il marxiano uomo onnilaterale.

È all'utopia che pensa Parinetto quando, sulla scorta dell'accezione alchemica, declina in senso dialettico il concetto di alienazione intesa come divenire altro da sé27. L'uomo onnilaterale, infatti, è un non-ancora-uomo, è «un concetto-progetto» 28 di critica delle situazioni alienate che egli vede prefigurato nel pensiero di Rousseau e nella sua concezione dello stato di natura: non un ideale nostalgico e bucolico, ma un modello critico del presente storico, un grimaldello potenziale per scardinare uno status quo che legittima le disuguaglianze socio-economiche spacciandole per condizioni naturali e immodificabili. Tuttavia, per Parinetto, lo stato di natura non è concepito tanto come un'ipotesi critico-metodologica quanto come un ideale utopico cui rivolgersi per costruire l'uomo onnilaterale, su cui insiste più di quanto non faccia lo stesso Marx, anche sulla scia della rilettura dei Deleuze-Guattari di Millepiani $^{29}$ e del Marcuse di Eros e civiltà: l'alienazione "positiva» del Contratto sociale come cessione delle libertà proprie di uno stato selvaggio a favore di un moi commun garante dei diritti prefigura l'integrazione democratica tra il personale e il politico e di conseguenza la condizione disalienata dell'uomo onnilaterale. Parinetto guarda dunque a Rousseau come a un precursore di Marx, sotto questo profilo ${ }^{30}$.

D'altronde, togliere l'alienazione è comunque possibile perché essa è solo «la forma storica di un'oggettivazione estraniata» ${ }^{31}$, per cui non è parte essenziale dell'uomo in quanto Gattungswesen, che si oggettiva con il lavoro. In questo contesto si possono collocare gli scritti giovanili sull'opera

\footnotetext{
${ }^{27}$ Un'accezione, questa, ripresa da Hegel. Cfr. L. Parinetto, Nostra signora dialettica, cit., p. 43. 28 Ivi, p. 48.

$29 \mathrm{Su}$ questo si veda soprattutto V. Verdesca, "Parinetto e Deleuze-Guattari: una conversazione", in M. Bellini (a cura di), Corpo e rivoluzione. Sulla filosofia di Luciano Parinetto, cit., pp. 99-118.

${ }^{30} \mathrm{Ha}$ analizzato questo aspetto M. Domina, "Natura umana e Ganzheit nell'opera di Luciano Parinetto", in M. Bellini (a cura di), Corpo e rivoluzione. Sulla filosofia di Luciano Parinetto, cit., pp. 67-82.

${ }^{31}$ L. Parinetto, Teorie dell'alienazione, cit., p. 153.
} 
verdiana $^{32}$, che Parinetto apprezza per intero e senza riserve, giacché vi legge il dispiegarsi della triade tematica alienazione-dialettica-utopia, la quale a sua volta si riassume nella denuncia della Trennung borghese tra il personale e il politico ${ }^{33}$. Parinetto riconosce che in Verdi la penetrazione nei sentimenti non è mai decadentistico compiacimento per il ripiegamento interiore, ma comprensione della complessità umana nella sua relazione con il più ampio quadro politico e sociale. Il via vai dialettico dal singolare all'universale deriva dal tentativo di riscattare l'alienazione, togliendo, in altri termini, la scissione tra l'individualità/diversità e l'alterità, che rappresenta la "norma", e non solo la legge, cui bisogna adeguarsi o sottomettersi. Pertanto la dialettica, qui concepita come andirivieni lessinghiano di TrennungVereinigung ${ }^{34}$, denunciando l'alienazione, apre alla prospettiva dell'utopia rivoluzionaria e questa consapevolezza rappresenta l'assunto di base delle riflessioni sulla stregoneria.

\section{Il capitale e la persecuzione delle streghe}

Punto di partenza per capirne il significato è Magia e ragione, dove tenendo conto delle opere di autori come Gian Rinaldo Carli, Bartolomeo Melchiori, Costantino Grimaldi, tra gli altri, Parinetto ricostruisce sul piano storico e culturale il riaccendersi quasi improvviso, nel periodo illuministico, della

\footnotetext{
32 Editi soprattutto su «L'eco di Brescia» e «La Verità», sono ora raccolti in L. Parinetto, Verdi e la rivoluzione. Alienazione e utopia nella musica verdiana, a cura di M. Bellini e G. Scaramuzza, Mimesis, Milano-Udine 2013. Su questo si vedano i saggi di R. Baldo, "Luciano Parinetto e la musica", in ivi, pp. 7-12 e di Scaramuzza, "Un amore di Parinetto", cit.

33 A proposito del Don Carlo, scrive per esempio che "Verdi non scinde l'individuo dal 'cittadino', l'uomo interiore dall'uomo politico; il tetro e geloso Filippo, per Verdi, è anche e soprattutto un re, perseguitato, anche nei momenti di più appartata meditazione, dalle preoccupazioni politiche» (L. Parinetto, "Giuseppe Verdi è un decadente?", in Verdi e la rivoluzione, cit., 42).

34 Del resto Parinetto non dà mai una definizione sistematica della dialettica: come scrive Alfredo Marini, «una configurazione ricorrente ma saltuaria, prettamente hegeliana, è sì quella che si concentra nel termine Aufhebung, ma nel suo svolgimento medio, il discorso veleggia sul profilo più basso e più fondamentale che si esprime nella coppia TrennungVereinigung". A. Marini, "Prefazione", in L. Parinetto, Nostra signora dialettica, cit., p. vii.
} 
discussione sulla caccia alle streghe che aveva insanguinato l'Europa moderna soprattutto a partire, ma non senza preamboli tragici, dalla diffusione del Malleus maleficarum dei domenicani Sprenger e Institoris del 1486, divenuto un vero e proprio manuale degli inquisitori nei secoli a venire.

La nuova ondata persecutoria è occasionata dalla pubblicazione del Congresso notturno delle Lammie di Girolamo Tartarotti nel $1749^{35}$ e Parinetto ne rileva la concomitanza con gli albori dell'età dei Lumi (il primo volume dell'Encyclopédie è del 1751), che segna, tra l'altro, il trionfo del cosmo galileiano-newtoniano e l'apogeo del paradigma meccanicistico della nuova scienza: alle essenze metafisiche e alle qualità della tradizione magicoalchemica propria del naturalismo umanistico, vengono a sostituirsi cause fisiche e quantità misurabili, che sanno spiegare la varietà dei fenomeni naturali con un rigore matematico estraneo al pensiero degli aristotelici. Eppure il passaggio dalla cultura animistica del periodo rinascimentale alla mentalità scientifica della prima età moderna non basta a estinguere la fede nella realtà del volo delle streghe.

Parinetto considera la simultaneità degli eventi e legge nella recrudescenza del pregiudizio stregonesco il tentativo fallito dell'illuminismo di espellere dalla cultura l'irrazionale, il diverso, il negativo, l'errore, che al contrario egli si propone di riqualificare offrendo una rilettura della dialettica in una peculiare chiave antropologica, dove l'irrazionale è tale se considerato in sé, ma mai può esserlo se inteso come espressione di una ragione diversa, non canonica, portatrice di un'apertura utopica.

Parinetto, infatti, vede nella stregoneria, sulla scia del Marx del Capitale, un'ideologia sovversiva dello status quo illuministico-borghese: come sottolinea nella prefazione alla nuova edizione del testo intitolata I Lumi e le

35 Di Tartarotti Parinetto aveva già tradotto i Cogitata circa strigas del 1749. Cfr. L. Parinetto, "Nascita del congresso notturno: i Cogitata circa strigas ed altri inediti tartarottiani", ACME. Annali della Facoltà di Lettere e Filosofia dell'Università degli studi di Milano, XXIV/1, 1971, pp. 71-96. 
streghe (1998), «Marx, infatti, nel primo libro del Capitale, connette la sparizione della persecuzione delle streghe all'incremento del processo di accumulazione del capitale bancario e nota che, essendo divenuta - dalla fine del '600 - la Banca d'Inghilterra «il serbatoio dei tesori metallici del paese e il centro di gravitazione di tutto il credito commerciale», «in Inghilterra proprio mentre si smetteva di bruciare le streghe, si cominciò a impiccare i falsificatori di banconote» ${ }^{36}$.

Alla persecuzione dei ribelli al vecchio dio biblico, i nuovi inquisitori del capitale andavano dunque sostituendo quella contro i recenti eretici, che, falsificando il nuovo dio-denaro, ne mettevano pericolosamente in forse la novella potenza» ${ }^{37}$.

Qui il legame tra stregoneria e capitale - o, meglio, tra la fine della caccia alle streghe e il trionfo del capitalismo finanziario, che identifica/crea novelle streghe da perseguitarsi - è ben messo in evidenza, ma è ancora in bozze, in Magia e ragione, l'esplicitazione della logica che sottende a tale processo inquisitorio (peraltro ben lontano dall'essere una mera proiezione, à la Freud, dei demonologi), e che, dato il riemergere di un antico pregiudizio ora funzionale alla logica di mercato, è una logica dialettica, il cui significato diverrà maturo negli anni a venire, quando Parinetto indagherà le origini storiche del fenomeno stregonesco.

È infatti nei primi anni '90, in testi come Solilunio (1991) o La traversata delle streghe nei nomi e nei luoghi (1993), che analizza documenti della prima età età moderna come testi di stregologi, bolle e brevi papali, resoconti di viaggiatori, comunque non senza la coscienza della loro scarsa attendibilità, rappresentando il solo punto di vista della griglia inquisitoria, ma anche perché la cultura delle streghe è solo orale e, quando trascritta, riflette le parole degli accusatori. In ogni caso le fonti servono a Parinetto per evitare

36 K. Marx, Il Capitale, introduzione di M. Dobb, tr. it. di D. Cantimori, Editori Riuniti, Roma 1964, Libro I, p. 818.

${ }^{37}$ L. Parinetto, I Lumi e le streghe, cit., p. 25. 
letture pregiudiziali che arrivino pure a ridurre la stregoneria a un epifenomeno folkloristico delle società tardo-feudali o persino a interpretarla in termini psicoanalitici: «occorre badare allo storico/specifico in cui dislocare ogni stregoneria, non correre il rischio di interpretarla ideologicamente, facendone un astratto regresso dalla cultura alla natura», quindi bisogna evitare di leggerla come una «negazione assoluta» della cultura, quando al contrario si configura piuttosto come la contestazione "determinata di una cultura socio/politica a sua volta non assoluta, ma determinata» ${ }^{38}$.

Ne La traversata delle streghe Parinetto, non senza il corredo di categorie marxiste, esplora la natura della relazione non casuale tra l'esplosione della caccia alle streghe nell'età moderna e l'emergere dell'economia capitalisticoborghese, sostenendo al riguardo che

le grandi persecuzioni delle streghe in Occidente non sarebbero affatto un orroroso lascito d'un affabulato 'buio' Medioevo, che inquina la luce del Rinascimento e gli albori dell'Età moderna, ma, invece, una consapevole applicazione di metodi politici di sterminio, in vista della dominazione, sperimentati con successo dal Potere durante le cruente vicende di assoggettamento dei popoli amerindi, da parte dei primi colonizzatori dell'America, ed esportati nella vecchia Europa, sempre in vista dell'imposizione del dominio. L'originario capitale, cioè, streghizza il Vecchio ed il Nuovo Mondo, non solo per dominare/annientare ogni tentativo di opposizione o di ribellione, ma, rinvenendo nel lavoro mal pagato (o addirittura forzato) degli indios (superstiti di un'immane carneficina) lo schema stesso mediante il quale avvierà le masse dei miserabili, dei mendicanti, dei diversi del Vecchio mondo sulla via del cosiddetto "libero mercato del libero lavoro", che sono il basamento stesso della valorizzazione. Sicché la strega (nella complessa accezione che assume il termine nei miei lavori) diventa inaspettatamente una delle figure originarie del cosiddetto 'libero' lavoratore del capitale! 39 ,

libero, cioè, di vendersi sul mercato concorrenziale del lavoro, ma d'altronde costretto a farlo per vivere. «Nel genocidio non v'è dunque solo lo sterminio militare, v'è quello, meno appariscente (perché quotidiano) ma non meno atroce, mediante lavoro (forzato). E si sa che il capitale comincia ad accumularsi sul basso prezzo del lavoro: il prezzo del lavoro di questi indios

${ }^{38}$ L. Parinetto, Solilunio. Erano donne le streghe?, prefazione di A. Agnoletto, A. Pellicani, Roma 1991, pp. 53, 52.

${ }^{39}$ L. Parinetto, La traversata delle streghe, cit., p. 8. 
era pressoché inesistente» ${ }^{40}$. «È dunque chiaro che v'è un rapporto fra fabbricazione di streghe, a scopo di sfruttamento lavorativo, e incremento del capitale, che sostituisce, al rogo, il lavoro forzato» ${ }^{41}$. La caccia alle streghe è quindi «uno stratagemma politico volto all'eliminazione delle diversità socio/politiche/religiose» ${ }^{42}$ e le streghe diventano dunque «il paradigma di ogni ceto che non accettava il mercato del lavoro» ${ }^{43}$.

La strega non rimanda dunque affatto a un generico rimosso ancestrale il cui ritorno farebbe regredire la civiltà a uno stadio primitivo e barbarico, ma è un fantasma reale, per ricorrere a un ossimoro dialettico nello stile parinettiano, che, con le sue facce multiformi, inquieta il capitalismo nascente perché rischia di destabilizzare i rapporti di forza grazie ai quali si realizza l'accumulazione di denaro. È proprio grazie agli studi storici che Parinetto smaschera la logica che sottostà al fenomeno della caccia alle streghe, al suo apparente eclissarsi agli inizi del XVIII secolo e al suo rigurgito in pieno illuminismo. Non è infatti il passaggio da una mentalità magico-animistica a una scientifica "ciò che toglie credibilità al volo delle streghe», ma anzi «è la logica del denaro (all'interno di una società che inizia ad essere ampiamente capitalistica) che scaccia la "logica" della teologia, della demonologia e della magia. È il denaro (come riconosce Shakespeare, teste Marx) che costringe gli opposti a baciarsi, che, cioè, introduce una logica dialettica sconosciuta ad epoche precedenti e per la quale, se, da un lato, va distruggendo il vecchio mondo di dio, del diavolo e delle streghe, dall'altro, se è suo interesse, contribuisce invece a rafforzarlo, quando (come succede durante la Conquista) streghizzazione coincide con valorizzazione!» ${ }^{4}$.

\footnotetext{
${ }^{40}$ Ivi, p. 131.

${ }^{41}$ Ivi, p. 133.

${ }^{42}$ L. Parinetto, "Introduzione", in Streghe e potere, cit., pp. 22-23.

${ }^{43}$ L. Parinetto, Streghe e politica, cit., p. 227.

${ }^{44}$ L. Parinetto, La traversata delle streghe, cit., p. 48.
} 


\section{Il diverso}

Ciò che emerge dalla ricerca di Parinetto riguardo ai moventi delle differenti carneficine è la necessità sotterranea dei poteri costituiti di disciplinare la corporeità: tale controllo è infatti orientato solo apparentemente a ossequiare ai divieti ecclesiastici, dunque allo scopo di sottrarre il corpo stesso alla sua riduzione a cosa; in realtà, dietro la maschera ideologica della colonizzazione o della conversione religiosa, si cela l'intento di uniformarlo al valore di scambio di tutte le altre merci, quindi valorizzando la sua utilizzabilità e sfruttabilità, proprio limitandone il valore d'uso potenzialmente rivoluzionario, un valore peraltro coestensivo anche al libero soddisfacimento delle pulsioni ${ }^{45}$.

In Solilunio, del 1991, Parinetto distingue tra al di qua e «al di là della griglia inquisitoria» ${ }^{46}$ per denunciare che è in particolare la "diversità sessuale» a sussistere come uno degli strati della surdeterminazione della stregoneria ${ }^{47}$. Ma in generale la strega è assimilabile al diverso tout court $^{48}$, in cui si comprendono infatti le donne, gli indios, gli ebrei, gli omosessuali, gli zingari, gli emarginati e tutte le categorie sociali escluse o comunque non integrate nel sistema capitalistico e che suscitano odio perché vengono etichettate come contro natura ${ }^{49}$, quando in realtà si pongano proprio contro

\footnotetext{
45 Precisa Nicoletta Poidimani che «il recupero parinettiano del basso, inteso in senso alchemico e rabelaisiano, va proprio in direzione del recupero del valore d'uso del corpo (e delle sue potenzialità) a fronte della scotomizzazione cui il modo di produzione capitalistico l'ha costretto. In questo quadro, il recupero, ad esempio, del culo e della merda - nominati come tali, senza eufemismi - si rivela essere un'arma affilata contro un sistema sociale che aliena le potenzialità del godimento anale nell'Analcharakter - tipico del tesaurizzatore - e che sublima nel denaro le potenzialità sovversive della merda e del corpo che la produce». N. Poidimani, "Luciano Parinetto: un precursore della Queer Theory?", in Corpo e rivoluzione in Marx, cit., p. 410.

${ }^{46}$ L. Parinetto, Solilunio, cit., p. 3.

47 Ivi, p. 248.

${ }^{48}$ Cfr. L. Parinetto, La traversata delle streghe, cit., p. 139.

${ }^{49}$ Cfr. L. Parinetto, Solilunio, cit., p. 199.
} 
i limiti che alla natura, e soprattutto alla natura umana, vengono imposti da una certa cultura.

Parinetto smaschera dunque l'ideologia che «istituisce l'equazione strega/donna»50, peraltro non avallata neppure dal Malleus che «non esclude affatto la partecipazione degli uomini alla stregoneria!»51; del resto, «se la stregoneria fosse solo un affare di donne, perché i succubi? „52. La persecuzione delle streghe non va quindi letta come una guerra tra i sessi, ma come la «strategia della normalità (in tutti i sensi, da quello economico e politico a quello religioso e non escluso quello sessuale) contro il diverso destabilizzante e inaccalappiabile» ${ }^{53}$ : in questo modo il pregiudizio sessista non sarebbe da rinvenirsi a monte della caccia alle streghe, ma a valle, come conseguenza dello stereotipo architettato ad arte per occultare le ragioni autentiche della carneficina.

Se poi si considera che tra le streghe figurano anche bambini, eretici, omosessuali, tra gli altri, è lecito inferire che «la strega sub specie aeternitatis non esista» ${ }^{54}$, anzi, per essenza è $«$ surdeterminata ${ }^{55}$, nel senso che non consiste in un'identità se non perché costruita in modo artificioso a partire dalle confessioni estorte con le violenze inflitte dall'Inquisizione. Citando il gesuita Spee, «è la tortura che fa la strega!»56 e, del resto, i meccanismi dei processi così istruiti sono tali «che $i$ giudici hanno sempre ragione! $\aleph^{57}$, quindi riescono a inchiodare la strega all'identità scomoda da eliminarsi attribuendole un nome di cui in realtà è portatore proprio l'inquisitore, «nella sua proiezione», nel momento stesso in cui lo pronuncia per etichettare la

\footnotetext{
${ }^{50}$ Ivi, p. 9.

51 Ivi, p. 74.

52 Ivi, p. 69.

${ }^{53}$ Ivi, p. 12.

54 Ivi, p. 10.

${ }^{55}$ L. Parinetto, La traversata delle streghe, cit., p. 19.

${ }^{56}$ L. Parinetto, Solilunio, cit., p. 215. Pertanto l'ammissione dei fatti di cui è accusata, peraltro sulla base di sospetti e delazioni, non risulta attendibile, come denuncerà anche Beccaria.

${ }^{57}$ Ivi, p. 221.
} 
vittima ${ }^{58}$. Ma «la strega è vagabonda per definizione», slittando «fra luoghi e nomi», come «dal femminile al maschile (e viceversa)», per cui «non vi è un ambito preciso in cui sia coglibile» se non per il tramite dello stigma con cui viene marchiata dall'inquisitore di turno ${ }^{59}$. Non consta di un'essenza, la sua natura è metamorfica, perciò sfuggevole, ma il suo essere inafferrabile, relegandola ai margini della società del capitale in cui non può trovare una collocazione stabile, la rende per essa minacciosa: il nuovo assetto economico prolifera grazie all'esclusione dei diversi, cioè dei poveri, dei mendicanti, dei contadini, dei proletari, per non parlare di stranieri, donne, omosessuali, ebrei ${ }^{60}$.

D'altronde la diversità rispetto alla norma costituita non è per Parinetto da emendarsi, anzi, la coscienza della diversità altro non è che una forma della coscienza di classe: l'emancipazione umana, il riscatto sociale, l'uomo onnilaterale, non possono realizzarsi grazie al riconoscimento della diversità, cioè all'integrazione nell'uguale. Già ne L'utopia del diavolo (1973) scriveva infatti che non è l'uguaglianza che va raggiunta per superare la dialettica servo-padrone alla base della società borghese, ma piuttosto va affermata la diversità inaccalappiabile e transeunte, e non per contrappunto, ma per far saltare i termini della relazione stessa: «l'eguaglianza cui si giunge dopo la rivoluzione (anche quella contro il capitale) è ancora una posizione per negazione, dipende ancora dalla diseguaglianza negata: vera nuova posizione sarebbe quella della diseguaglianza disalienata» ${ }^{61}$. Riconoscimento della diversità, quindi, non significa toglimento dell'alienazione, ma suo sigillo,

\footnotetext{
${ }^{58}$ L. Parinetto, La traversata delle streghe, cit., p. 20.

${ }^{59}$ Ivi, p. 19.

${ }^{60}$ Un'ampia disamina di queste categorie "oziose" si trova in Streghe e politica, cit., pp. 213 sgg., ne La traversata delle streghe, cit., e in L. Parinetto, L. Sichirollo, Marx s Shylock. Kant, Hegel, Marx e il mondo ebraico, Unicopli, Milano 1982.

${ }^{61}$ L. Parinetto, Corpo e rivoluzione in Marx, cit., p. 181.
} 
volto a consacrare la perdita del «pungiglione contestatore» ${ }^{2}$ della diversità medesima ${ }^{63}$.

Il diverso, se riconosciuto invece "come diverso» ${ }^{44}$, non è fatto rientrare entro i confini della norma; anzi, è colui che, resistendo all'omologazione all'identico e rivendicando la propria diversità, può essere rivoluzionario, quindi portatore di utopie ${ }^{65}$. Riecheggiare, dunque, il motto sessantottesco femminista, ma fatto proprio anche da altre minoranze come gli omosessuali - per cui il personale è politico è ormai da ritenersi «una battaglia del tutto interna alla società borghese» perché «è riconoscimento non di ciò che uno è, ma dell'atomo di una serie» nella quale si identifica ${ }^{66}$. In questo senso, il «mito dell'emancipazione» "ha castrato la rivoluzione»67: emanciparsi, in un'ottica "cittadinista" estranea a Parinetto, vuol dire integrarsi, riconoscersi identici, nella forma, agli altri membri della società civile; significa, per ricorrere a note espressioni marxiane, illudersi che il cielo dello Stato si realizzi hic et nunc attraverso le regole di una democrazia formale e il principio di tolleranza. È in questo modo che il diverso potrebbe diventare politico.

Ma ormai è tramontato il tempo per rivendicare l'identità tra il personale e il politico, giacché il politico si incarna ormai solo nel borghese e nelle sue alienazioni: la delusione del '68 è ancora cocente - la critica di Pasolini ne è il vessillo - e il marxismo italiano delle istituzioni comincia a involvere in quel revisionismo che lo avvicinerà al compromesso storico, del quale, nella premessa a Ritagli, volume che, confezionato in modo artigianale, raccoglie le

\footnotetext{
62 L. Parinetto, Faust e Marx, cit., p. 206. Per esempio, quando, infatti, «il cosiddetto omosessuale rinuncia alla propria ineffabile diversità e si omologa nella serializzazione del riconoscimento, proprio così accetta il marchio da parte dello stato e della società borghese». Ivi, p. 205.

${ }^{63}$ Cfr. N. Poidimani, "Diversità dei diversi", in N. Poidimani (a cura di), Luciano Parinetto. L'utopia di un eretico, cit., pp. 61-70.

${ }^{64}$ L. Parinetto, Faust e Marx, cit., p. 201.

65 «Diversità del diverso» è peraltro il titolo del quarto frammento/capitolo di Faust e Marx, cit.

66 Ivi, p. 205.

${ }^{67}$ Ivi, p. 211.
} 
fotocopie di tutti gli articoli pubblicati sui quotidiani bresciani ${ }^{68}$, Parinetto dà un giudizio tranchant, denunciando oltretutto la compromissione del P.C.I. con la destra fascista nella "giustificazione del finanziamento al M.S.I.», elargito addirittura «dopo la strage di Brescia» ${ }^{69}$ in Piazza della Loggia.

Nel 1978, anno del compromesso storico tra D.C. e P.C.I., Parinetto inasprisce la sua diffidenza per la vita politica del Paese e ripensa, pertanto, anche la natura della relazione tra il privato e il pubblico, tra le scelte etiche personali e la cornice collettiva in cui si collocano. Ne scrive in proposito in un breve contributo saggistico dal titolo Lettera non politica sullinquisitore, destinato a un volume collettaneo sull'opera verdiana, curato da Mario Lussignoli cui è indirizzata la lettera, e pubblicato dalla Fondazione CalzariTrebeschi di Brescia in occasione dell'allestimento, presso il Teatro Grande, del Don Carlo di Ronconi ${ }^{70}$. Parinetto, tornando per la prima e ultima volta su Verdi, rettifica quanto aveva sostenuto proprio negli articoli verdiani giovanili, dato che «non io, ma la storia», ha posto «in luce critica, quasi che quella prospettiva» volta ad affermare l'identità tra il personale e il politico «sia da rovesciare»: oggi, infatti, il politico è la "più squallida figura di ALIENAZIONE che ci sia dato conoscere», pertanto il personale semmai deve avviarsi «a diventare non più politico» ${ }^{71}$. Anzi, «il diverso veramente tale emerge come l'opposto (non dialettico) del politico» ${ }^{72}$.

Alla luce dell'inedito corso storico della sinistra italiana che si avvia allo strappo di Berlinguer con l'Unione Sovietica, Parinetto rifiuta di accomodarsi alla prospettiva integrazionista, in quanto, coerente con l'ideologia fondante del compromesso storico, è intuita per ciò che sarà, cioè la padrina della "terza

\footnotetext{
68 Il volume è custodito presso la Fondazione Morcelli-Repossi di Chiari, in provincia di Brescia, nel Fondo Parinetto.

${ }^{69}$ L. Parinetto, "Premessa a Ritagli", in Verdi e la rivoluzione, cit., p. 7.

${ }^{70}$ L. Parinetto, "Lettera non politica sull'inquisitore", in Verdi e la rivoluzione, cit., pp. 97105; già in M. Lussignoli (a cura di), "Don Carlo" di Giuseppe Verdi. Materiale storico-critico per l'audizione, Fondazione Calzari Trebeschi, Brescia 1978, pp. 187-200.

${ }^{71}$ Ivi, p. 98.

${ }^{72}$ L. Parinetto, Faust e Marx, cit., pp. 211-212.
} 
via" socialdemocratica, stigmatizzata come un'Aufhebung pacificante. Per Parinetto, l'integrazione sterilizza la dialettica rivoluzionaria perché toglie la contraddizione: semplicemente riconosce l'alterità, assimilandola allo status quo sotto le false spoglie dell'emancipazione. L'integrazione è una semplice posizione per negazione, pertanto dipende da ciò che nega e mantiene i limiti posti dalla questione, di conseguenza incarna un'ideologia conservatrice che tollera il dissenso assimilandolo a sé, ovvero di fatto reprimendolo grazie alla concessione, solo in apparenza democratica, di uno spazio espressivo interno al sistema. L'integrazione non sollecita il cambiamento, ma cerca il riconoscimento, appunto. Perché, al contrario, si metta in atto un cambiamento effettivo e radicale, bisogna far saltare la relazione stessa togliendone i termini e in questo consiste la dialettica rivoluzionaria, cui Parinetto resta fedele in tutti i suoi scritti.

\section{Nota bibliografica}

BALDO, Renzo, "Luciano Parinetto e la musica", in L. Parinetto, Verdi e la rivoluzione. Alienazione e utopia nella musica verdiana, a cura di M. Bellini e G. Scaramuzza, Mimesis, Milano-Udine 2013, pp. 7-12.

BELLINI, Manuele (a cura di), Corpo e rivoluzione. Sulla filosofia di Luciano Parinetto, Mimesis, Milano-Udine 2012.

-, "Il Fondo Parinetto tra marxismo, stregoneria e alchimia", in F. Formenti (a cura di), La Biblioteca Morcelliana nel bicentenario (1817-2017). Studi e ricerche, Morcelliana, Brescia 2017, pp. 257-262.

-, Dialettica del diverso. Marxismo e antropologia in Luciano Parinetto, Mimesis, Milano-Udine 2018.

BORSO, Dario, "Introduzione" a M. Dal Pra, Il pensiero filosofico di Marx, ShaKe, Milano 2011, pp. 5-11. 
—, "Prefazione", in L. Parinetto, Teorie dell'alienazione. Hegel, Feuerbach, Marx (1968), a cura di D. Borso, ShaKe, Milano 2012, pp. 7-8.

DAL PRA, Mario, La dialettica in Marx. Dagli scritti giovanili all'“Introduzione alla critica dell'economia politica”, Laterza, Bari 1965.

DOMINA, Mario, "Natura umana e Ganzheit nell'opera di Luciano Parinetto", in M. Bellini (a cura di), Corpo e rivoluzione. Sulla filosofia di Luciano Parinetto, Mimesis, Milano 2012, pp. 67-82.

ERACLITO, Fuoco non fuoco. Tutti i frammenti del filosofo di Efeso, a cura di L. Parinetto, Mimesis, Milano 1994.

FERGNANI, Franco, Alienazione e oggettivazione nella problematica del pensiero moderno e contemporaneo, Cuem, Milano s.d. ma presumibilmente 1972.

FRANZINI, Elio, "Utopie", in N. Poidimani (a cura di), Luciano Parinetto. L’utopia di un eretico, Mimesis, Milano 2005, pp. 39-46.

GALLI, Giorgio, "Solilunio tra streghe e alchimisti", in N. Poidimani (a cura di), Luciano Parinetto. L’utopia di un eretico, Mimesis, Milano 2005, pp. 55-60.

MANGANO, Attilio, "Il pensiero di Luciano Parinetto: un marxismo magico?", in N. Poidimani (a cura di), Luciano Parinetto. L'utopia di un eretico, Mimesis, Milano 2005, pp. 33-38.

PARINETTO, Luciano, Teorie dell'alienazione. Hegel, Feuerbach, Marx (1968), a cura di D. Borso, ShaKe, Milano 2012.

—, "Sul noviziato feuerbachiano di Marx", Utopia, luglio 1971, pp. 7-14.

—, "La religione nel 'Capitale”, Utopia, agosto 1971, pp. 4-10. 
—, “Karl Marx e Moses Hess”, Utopia, settembre-ottobre 1971, pp. 11-18.

_, "Sulla forma della religione in Marx", Utopia, novembre 1971, pp. 7-13.

_, “Tre immagini dell'intellettuale Marx”, Utopia, dicembre 1971, pp. 7-14.

_, "Alienazione e utopia in Rousseau”, Utopia, marzo 1972, pp. 8-16.

—, "Alienazione e utopia in Lessing", Utopia, maggio 1972, pp. 3-8; parte II, giugno 1972, pp. 8-14.

—, "Antimacchinismo e umanità in Herder", Utopia, settembre 1972, pp. 3-10.

—, "Ernst Bloch: un teologo?", Utopia, ottobre 1972, pp. 18-25.

—, "Corpo e rivoluzione in Marx", Utopia, gennaio 1973, pp. 3-12.

—, "L’ateismo di Marx: una leggenda", Utopia, marzo 1973, pp. 3-12.

—, "Morte e utopia", Utopia, giugno 1973, pp. 3-13.

—, "Schiller segreto", Utopia, settembre 1973, pp. 3-10.

—, "Gioco e rivoluzione in Schiller", Utopia, ottobre 1973, pp. 3-13.

—, "L'utopia del diavolo. Egualitarismo e transessualità", Utopia, dicembre 1973, pp. 19-28.

-, Magia e ragione. Una polemica sulle streghe in Italia intorno al 1750, La Nuova Italia, Firenze 1974 (riedito col titolo I Lumi e le streghe, Colibrì, Milano 1998).

—, Né dio né capitale. Marx, marxismo, religione, Moizzi Contemporanea, Milano 1976. 
-, Corpo e rivoluzione in Marx. Morte, diavolo, analità (1977), Mimesis, Milano-Udine 2015.

—, Streghe e politica. Dal rinascimento italiano a Montaigne, da Bodine a Naudé, prefazione di G. Galli, I.P.L., 1983.

—, Faust e Marx. Metafore alchemiche e critica dell'economia politica. Satura inconclusiva non scientifica (1989), Mimesis, Milano 2004.

—, Alchimia e utopia (1990), Mimesis, Milano 2004.

-, Nostra signora dialettica. Unificazione e scissione in Lessing, Herder, Schiller, prefazione di A. Marini, A. Pellicani, Roma 1991.

—, Solilunio. Erano donne le streghe?, prefazione di A. Agnoletto, A. Pellicani, Roma 1991.

—, La traversata delle streghe nei nomi e nei luoghi e altri saggi (1993), Colibrì, Milano 1997.

—, Il ritorno del diavolo, Mimesis, Milano 1996.

—, "Introduzione", in Streghe e potere. Il capitale e la persecuzione dei diversi, Rusconi, Milano 1998, pp. 7-25.

—, La rivolta del diavolo. Müntzer, Lutero e la rivolta dei contadini in Germani e altri saggi, Rusconi, Milano 1999.

—, Verdi e la rivoluzione. Alienazione e utopia nella musica verdiana, a cura di M. Bellini, G. Scaramuzza, Mimesis, Milano-Udine 2013.

PARINETTO, Luciano, SICHIROLLO, Livio, Marx e Shylock. Kant, Hegel, Marx e il mondo ebraico, Unicopli, Milano 1982. 
POIDIMANI, Nicoletta, "Diversità dei diversi", in N. Poidimani (a cura di), Luciano Parinetto. L’utopia di un eretico, Mimesis, Milano 2005, pp. 61-70.

- (a cura di), Luciano Parinetto. L'utopia di un eretico, Mimesis, Milano 2005.

—, "Luciano Parinetto: un precursore della Queer Theory?", in L. Parinetto, Corpo e rivoluzione in Marx. Morte, diavolo, analità (1977), Mimesis, Milano-Udine 2015, pp. 403-412.

RAMBALDI, Enrico I., "Gli insegnamenti filosofici nella Facoltà di Lettere (1924-1968)", in E. Brambilla, M. G. di Renzo Villata (a cura di), Per una storia dell'Università di Milano, CLUEB, Bologna 2008.

SCARAMUZZA, Gabriele, "Un amore di Parinetto", in Il brutto all'Opera. L'emancipazione del negativo nel teatro di Giuseppe Verdi, Mimesis, Milano-Udine 2013, pp. 157-181.

VERDESCA, Viviana, "Parinetto e Deleuze-Guattari: una conversazione", in M. Bellini (a cura di), Corpo e rivoluzione. Sulla filosofia di Luciano Parinetto, Mimesis, Milano 2012, pp. 99-118. 